\title{
Design and structural characterization of a bivalent MAP kinase inhibitor
}

Bernhard C. Lechtenberg*, Peter D. Mace, E. Hampton Sessions, Robert Williamson, Romain Stalder, Yann Wallez, Gregory P. Roth, Stefan J. Riedl and Elena B. Pasquale

*Sanford Burnham Prebys Medical Discovery Institute, La Jolla, USA

blechtenberg@sbpdiscovery.org

The RAS-RAF-MEK-ERK mitogen-activated protein kinase (MAPK) pathway controls a large variety of physiological processes and accordingly is linked to many diseases, especially cancers. Activating mutations in RAS and BRAF are found in about $40 \%$ of all cancers. Thus, the pathway is a major drug target against cancer and several kinase inhibitors mainly targeting RAF and MEK are in the clinic. Although the initial response to these drugs can be dramatic, development of drug resistance is a major challenge. Combination therapy of two inhibitors targeting both RAF and MEK can delay, but not stop the occurrence of resistance. Importantly, most resistance mechanisms still rely on activation of the downstream effector kinase ERK and thus drug development focus has shifted to directly inhibit ERK. We designed a set of bivalent ERK inhibitors that combine a small molecule drug that targets the ATP pocket with ERK-specific peptides, which bind to a protein-protein interaction site on ERK. To obtain better insight into their binding details we first generated crystal of phosphorylated (active) ERK with the ATP analog AMP-PCP. This allowed us to develop a soaking approach exchanging AMP-PCP with different bivalent inhibitors for successful structure solution. These structures confirmed the bivalent binding mode of the inhibitors. Additional kinase activity and binding studies reveal that the bivalent inhibitors have vastly improved potency towards ERK and high specificity towards ERK compared to other kinases. However, unexpectedly, they also are able to inhibit p38 and JNK, two MAPKs, which contain a similar peptide-binding site, despite reports that emphasize the peptide specificity of the protein-protein interaction site endemic to ERK. This observation implicates that testing inhibitor specificity is critical when designing bivalent inhibitors. 